\title{
Prognostic Ability of Practitioners of Traditional Arabic Medicine: Comparison with Western Methods Through a Relative Patient Progress Scale
}

\section{Bertrand Graz}

Faculty of Medicine, University of Lausanne, Switzerland

\begin{abstract}
The ancient Greek medical theory based on balance or imbalance of humors disappeared in the western world, but does survive elsewhere. Is this survival related to a certain degree of health care efficiency? We explored this hypothesis through a study of classical Greco-Arab medicine in Mauritania. Modern general practitioners evaluated the safety and effectiveness of classical Arabic medicine in a Mauritanian traditional clinic, with a prognosis/follow-up method allowing the following comparisons: (i) actual patient progress (clinical outcome) compared with what the traditional 'tabib' had anticipated (= prognostic ability) and (ii) patient progress compared with what could be hoped for if the patient were treated by a modern physician in the same neighborhood. The practice appeared fairly safe and, on average, clinical outcome was similar to what could be expected with modern medicine. In some cases, patient progress was better than expected. The ability to correctly predict an individual's clinical outcome did not seem to be better along modern or Greco-Arab theories. Weekly joint meetings (modern and traditional practitioners) were spontaneously organized with a modern health centre in the neighborhood. Practitioners of a different medical system can predict patient progress. For the patient, avoiding false expectations with health care and ensuring appropriate referral may be the most important. Prognosis and outcome studies such as the one presented here may help to develop institutions where patients find support in making their choices, not only among several treatment options, but also among several medical systems.
\end{abstract}

Keywords: Classical Arabic medicine/Greco-Arab medicine - medical theory - outcome prognosis - relativism

\section{Introduction}

In a clinic near Nouakchott, Mauritania, two practitioners, brother and sister, hold their consultations together on a carpet in a cool adobe house. They are surrounded by students (who also happen to be their nephews). The dispensary in the next room is filled with rough plant and mineral products, mostly gathered in the nearby area, some of them imported from far away, from Syria ('gum tragacanth' from Astragalus gummifer

For reprints and all correspondence: Bertrand Graz, Faculty of Medicine, University of Lausanne, Switzerland.

E-mail: bertrand.graz@chuv.ch
Labill.) or the Greek island of Chios ('gum mastic' from Pistacia lentiscus L.) (1). In the sandy courtyard, Bedouin tents are pitched, where in-patients live with their families who help with their care. Contrary to many traditional medical systems, there is no secret here, since all diagnostic procedures and treatments are described in the books kept on a small shelf in the consultation room.

Systematized Arabic medicine (AM), with its roots in the Greek Hippocratic tradition, owes much to the contributions of Avicenna (Ibn Sinna), soon after 1000 (of our common era). His followers worked in a huge cultural area stretching from Persia and the Middle East to the Iberic Peninsula (Andalusia and Spain), where medical schools flourished during the 11 and 12 th centuries. 
Medical textbooks from that time (the most famous are probably the 'Canon' and the 'Poem of Medicine', both by Avicenna) were to constitute enduring references of medicine for centuries, in the East and in the West. Its basic theory, based on the concepts of temperaments and balance or imbalance of humors and combinations of temperature and humidity, remained a standard approach in the West until the 18th century.

Today, in Mauritania, classical AM is used by large parts of the Mauritanian population, in all socioeconomic strata (2). Families of practitioners belong to the cultural elite. Although it has similarities with Indian and Pakistani 'Unani' medicine, it has been adapted to local conditions. Our objective was to conduct a rapid assessment of the AM practiced in a Mauritanian traditional clinic.

\section{Method}

Patient progress with classical AM (170 consecutive patients, with no exclusion) was assessed by modern GPs (General Practitioners) from the Swiss Cooperation Agency and Médecins du Monde-Switzerland, with a relative patient progress scale (Figs 1 and 2) allowing the following comparisons: (i) actual patient progress (clinical outcome) compared with what the TPs (traditional practitioners) had anticipated (= prognostic ability) and (ii) patient progress compared with what could be hoped for if the patient were treated by a modern physician in the same neighborhood.

The chosen method was proposed 20 years ago by the Nobel Prize winning physicist Richard Feinman and the oncologist Lucien Israel for assessment of complex and individualized cancer treatments (3). However, to our knowledge, this study is first to use this method. This method relies on the following rationale: before a patient is treated with a new or unknown treatment ('alternative treatment'), experienced practitioners in conventional medicine can make a clinical prognosis on how the

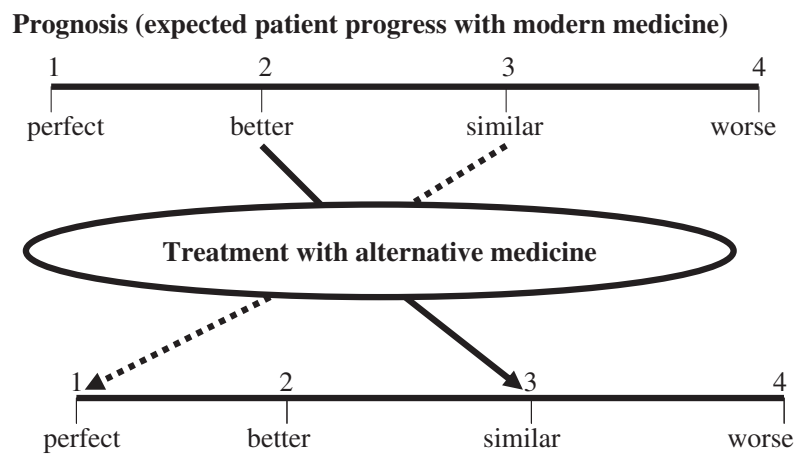

Outcome (observed patient progress)

Figure 1. The 4-point relative scale used for comparison of prognosis and outcome. In this study, alternative medicine was classical AM. patient would be likely to progress with the known, standard treatment (expected clinical course). Their prognosis is then compared with the observed progress under the alternative treatment (Figs 1 and 2).

Experienced GPs predicted course of disease (= GP prognosis) is used here as the reference because it lies at the very base of academic prescription practices: when a GP proposes a given treatment rather than another, he/she does this because he/she makes the prediction that patient progress will be better with this given treatment. More precisely, in today's 'evidence-based medicine' (EBM), the rationale is often not a logical deduction from a knowledge of pathogenic mechanisms. What EBM provides, is a risk-and-benefit ratio between different treatment options. Results of randomized controlled trials are a comparison of outcomes with various treatments given to similar patients. Following statistical rules, medical prescription is based on the claim that, like meteorologists, modern practitioners can be correct in their predictions more often than if they counted on chance only. In other words, GP prediction is the standard here because if it could not provide data of acceptable reliability, the whole modern biomedical prescription system would also lack reliability (and, indeed, in this hypothetical case, it would also lack an important criterion for being considered scientific, because the ability to use observations and theory to correctly predict subsequent events is a key element of science). The method used here is called prognosis/follow-up (4).

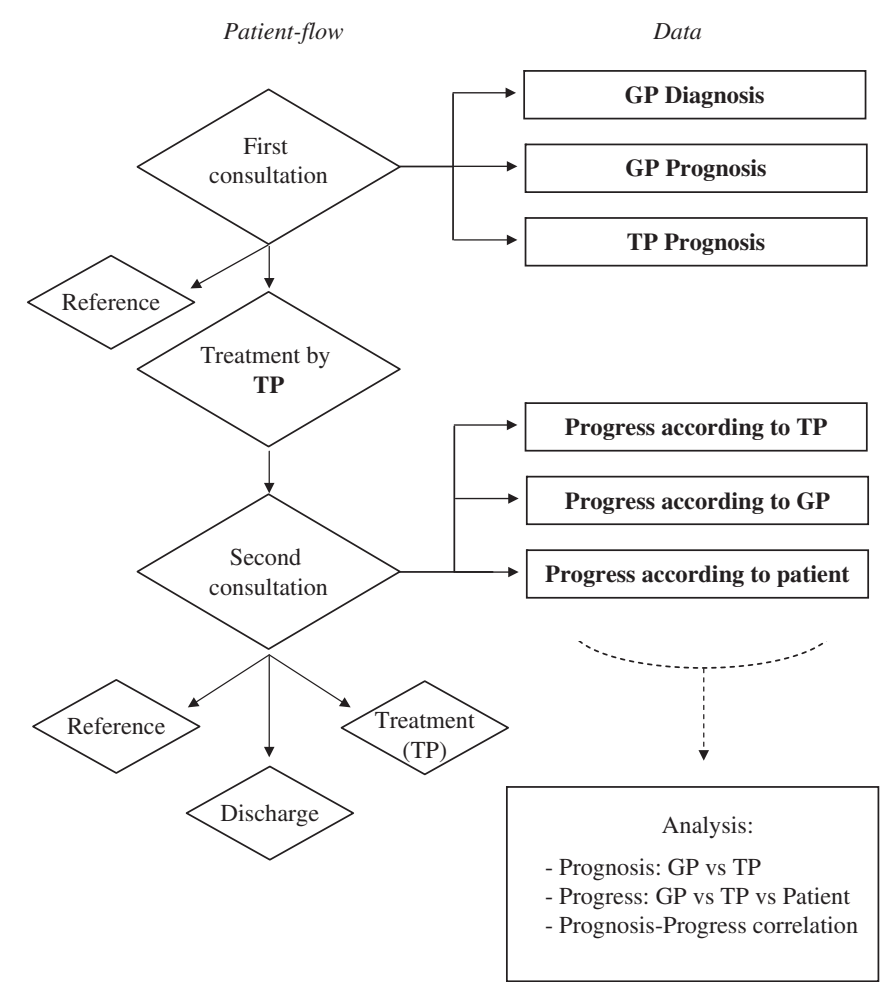

Figure 2. Structure of the prognosis-based method. 
Since the research process was based on a simple attendance, with no intervention nor special patient examination or observation, approval from an ethical committee did not come into consideration. Every patient consulting the TPs was asked whether a GP could attend the consultation; the proposal was welcomed by each of the 170 consecutive patients seen.

Data were analyzed with Epi-Info and Stata software. Health problems were manually classified. Homogeneity between patients seen versus lost to follow-up was studied with the Fisher's exact test. Degrees of agreement and differences between raters were analyzed, respectively, with the $\kappa$-statistic (weighted $\kappa=0.5$ for 1 rank discordance and 0 for more) and the Wilcoxon signed-rank test. TP prognosis ability was studied as a key-factor for making correct decisions on whether a particular patient would be better off with modern or classical AM (referral appropriateness).

\section{Results}

\section{Population and Morbidity: Tropical without 'Tropical Diseases'}

There were 170 patients, 74 females, 96 males, aged from 3 months to 80 years old (mean 38-years old); $13 \%$ were under 20 -years old and $22 \%$ above 50 . Patients presented mainly with chronic and congenital disorders, mental problems, functional and terminal diseases. GP diagnoses were very diverse, ranging from sequelae of perinatal anoxia to terminal cancer, with a high frequency of intestinal and liver problems.

\section{First Consultation: Advising Diet, Medicinal Plants or Referral}

A patient progress toward a 'perfect' or 'better' state was predicted by GPs in $80 \%$ of the cases, by TPs in $83.5 \%$ and by both in $69.4 \%$. Agreement over expected patient progress was relatively low, with TPs giving more optimistic prognoses than GPs (GP-TP $\kappa=0.2$, signedrank $P=0.006$ ). Among six patients judged by GPs as showing signs of danger, referral was proposed by GPs in one case only; others received a GP prescription (three prescriptions - the only ones during the study) or TP treatment; two of them had terminal cancer, two had an infectious disease, one heart failure and one unexplained shoulder dysfunction.

Referral to the modern health system was proposed by the GPs for eight patients $(4.5 \%)$ : two were actually referred by the TPs (plus one without GP advice), six were not referred (all of them apparently by patient decision, one showing signs of danger according to GPs). A laboratory test was ordered by GPs for 15 patients $(8.8 \%)$ and $\mathrm{X}$-rays in six cases.
TP main treatment was a traditional drug in half of the cases (85 cases), a special diet for 66 patients $(39 \%)$ and minor surgery in 10 cases $(6 \%)$ - others and no answers: nine cases, $5 \%$.

\section{Follow-up: As Expected in a GP Practice}

Follow-up was obtained for 113 patients (66\%). Patients lost to follow-up tended to have more indices of severity [4/57 with signs of danger against $2 / 110$ in patients seen at follow-up $(P=0.18)$; prognosis 'worse' in $5 / 57$, versus 2/113 $(P=0.04)]$.

TP prescriptions had been reportedly taken by all but 18 patients. Lack of money was the main reason for noncompliance (five cases), then 'refusal to eat' (three cases). No serious side effect was recorded. A modern treatment had been used in four cases.

At follow-up, patient progress was judged as 'perfect' or 'better' in most cases (80,90 and 90\% according to GPs, TPs and patient, respectively). Five deaths were reported, 3/ 5 before follow-up, 4/5 among patients staying near the clinic. Signs of imminent danger had been noted by GPs in one case (a patient suffering from terminal esophageal carcinoma, for whom both GPs and TPs agreed on palliative care at home). Concerning other reported deaths, fatal outcome had not been anticipated, neither by GPs nor by TPs; for these, GP diagnoses had been (without signs of imminent danger): cirrhosis, pancreatitis, hypertensive heart disease and chronic constipation, the latter being deceased ( 9 days after the consultation) from intestinal occlusion in the National (modern) Hospital.

For assessment of patient progress, inter-rater agreement was as shown in Table 1. In cases of discordance between TPs and GPs, TPs judged patient's progress more favorably than the GPs (in 30 cases, i.e. $27 \%$ of cases versus 1 case of more favorable judgment by GPs$P<0.001)$. Patient rating of his/her own progress was, between GP and TP, closer to GP $(P<0.02$ for both).

Progress with TP treatment, according to GPs, appears similar to what GPs had expected, had the patient been treated with standard medicine (Table 2). Overall, TPs judged patient progress as better than expected $(P=0.008$; patient progress judged as similar to what had been expected in 63 cases, better than expected in 35 cases and not as good as expected in 16 cases).

Table 1. Patient progress (patient status at follow-up), as compared with status at first consultation on relative patient progress scale

\begin{tabular}{lll}
\hline & Agreement $(\%)$ & $\kappa$ \\
\hline GP and TP & 79.2 & 0.50 \\
GP and patient & 86.6 & 0.63 \\
TP and patient & 84.7 & 0.57 \\
\hline
\end{tabular}

Comparison of judgments by general practitioner (GP), traditional practitioner (TP) and the patient him/her-self. $N=113$. 
Table 2. Results from the Mauritanian experience: Prognosis and Follow-up of patients treated with AM

\begin{tabular}{llllll}
\hline \multirow{2}{*}{$\begin{array}{l}\text { Expected } \\
\text { Progress }\end{array}$} & \multicolumn{2}{l}{ Observed Progress } & $\begin{array}{l}\text { Expected } \\
\text { Progress } \\
\text { Total }\end{array}$ \\
\cline { 2 - 5 } & Worse & Similar & Better & Perfect & Potect \\
\hline Perfect & 2 & 9 & 12 & 20 & 43 \\
Better & 1 & 7 & 32 & 14 & 44 \\
Similar & 0 & 2 & 9 & 3 & 14 \\
Worse & 1 & 1 & 0 & 0 & 2 \\
Observed & 4 & 19 & 53 & 37 & \\
Progress Total & & & & & \\
\hline
\end{tabular}

Sign-rank test: $P=0.38$.

Observed progress (outcome) of 113 patients treated by classical Arabic medicine, compared to expected progress (prognosis) with modern medicine (both assessed by general practitioners). Individual prognosis and outcome were determined for every patient; summary results are shown here.

One example of a patient progress better than expected was a 15 -year-old boy with a bone infection for which the nearby modern hospital had proposed amputation: after 2 weeks of local administration of Commiphora africana powder ('lembarka' i.e. 'the blessed'), the patient was able to walk, run and play football.

\section{Unexpected Results: Traditional and Modern Practitioners Start to Talk}

Weekly joint meetings (modern and traditional practitioners) were spontaneously organized with a modern health center in the neighborhood. Patients with difficult problems were presented there and appropriate referral (in both directions, from or to traditional AM) was discussed.

\section{Discussion}

The studied AM practice appeared fairly safe and, in many chronic conditions, local practitioners performed similarly to what can be expected with modern medicine. In some cases, patient progress was better than expected.

\section{A New Way to Assess Alternative Medicine with very Individualized Treatments}

Concerning the method used, this pilot project showed that the prognosis/follow-up method can be recommended, when local pre-tests are conclusive and conventional research methods impracticable for assessment of traditional or complementary health care. The process of making a prognosis was not studied; for this process, medical theory may not be as important as experience. GP prognosis was taken as the gold standard, because our modern prescription system is based on results from controlled trials, i.e. it does not rely on medical theory but on experiment leading to differential prognoses. If the general practitioner's predictions could not provide fairly reliable data, then our whole modern medicine could not be considered reliable either. This is so because, when caring for a patient, we need to envisage all possible outcomes (i.e. make predictions) with different therapeutic options ( $=$ results from comparative clinical studies), and then (with the patient) choose one.

The method allows selection, for further studies, of conditions for which recorded outcomes were better than expected. In addition to this selection process, testing a whole system of medicine could be used to show the global quality of care offered by an individual practitioner or in a given setting of alternative medicine.

About judgment on patient progress, our results can be compared with those obtained in Sweden, where professional ratings of health (on a 5-point graded scale) and self-rated health coincided in $\sim 60 \%$ of the cases, with a correlation of 0.45 (5). The present results, with almost $80 \%$ agreement between GP and TP judgements on patient progress, and higher for agreement between patient and GPs - or patient and TPs - exceeds those of the Swedish study. This may be due to the relative nature of the scale used here, where GPs, TPs and patients were requested to estimate a level of improvement, which might have made it easier to find a common language with close judgements. In a future study using a prognosis-based method, it might be appropriate to also record patient self-assessed health at first consultation, since it was found a significant predictor of functioning and mortality (6). It would also be useful to study inter-GP differences in prognosis ability, a realm that has not been often studied so far, except with critically ill patients, where it was found that different physicians frequently disagree substantially about patient prognoses (7).

Limitations of this study were primarily embedded in the method itself: the rating system is relatively crude (as compared to usual 10 degrees or visual analogue scales). We cannot know what part of observed differences between GP and TP ratings of patient progress are due to professional divergences, culturally or individually skewed understandings of the used scale - and what part represents a bias due to systematic desire to rate clinical observations up or down. The risk that patients lost to follow-up, if seen again, could have led to different results is real but was estimated fairly low, because we observed that patients did not hesitate to openly complain against TPs when anything seemed questionable to them, and important events such as fatal outcome appeared to be reported for patients not seen at follow-up.

Generalization of results requires great caution, because the studied setting is considered as one of the best in Mauritania and certainly does not reflect the average quality of traditional health care in the area. However, since the practice of classical AM is based on a written 
medical system, there may be a high level of homogeneity of health care (as compared to orally transmitted systems), and within a large cultural area that ranges (medically speaking) from Mauritania to India and Bangladesh.

\section{Beyond Theory: Experience and Effectiveness}

The results of this study can be partially explained by the 'case-mix': in the modern health center serving the same community, the most frequent diagnoses are infant and childhood diarrhoea, pneumonia and tooth decay. In the classical Arabic clinic, patients present with chronic and congenital disorders, mental problems, functional and terminal diseases; these diagnoses represent troubles for which modern medicine usually offers far from a miracle, especially in settings where the means are very limited. Patients appear to perform their own self-referral with very few errors, in view of the small proportion of referrals proposed by the GPs $(4.5 \%$ of all patients). Finally, we cannot exclude that AM has an intrinsic effectiveness in some cases, which would mean that good results can be obtained with a theory that is usually considered out of date by health care professionals in modern settings. If TP theory is wrong, how do TPs achieve both prognosis and clinical effectiveness? In consistency with today's tendency to use experience rather than theory as the base for 'evidence-based' medical practice, an answer could be: In the classical AM clinic practitioners achieve prognosis and clinical effectiveness because they rely on $>2000$ years of experience. If experience is the main factor of prognosis ability, then theory (whatever the medical system) would appear as benign aid for the medical practitioner, a sort of mnemonic tool with limited heuristic potential.

This study raises the question of scientific relativism: in natural sciences, what distinguishes a good scientific theory is that it makes correct predictions. In medicine, a good theory of disease should lead to a correct prognosis (patient anticipated progress if using a given treatment). In this study, predictions of TPs appeared of fair precision. What does that mean?

Is it possible that, with completely different assumptions about how human beings function, some therapeutic results might be comparable to those obtained with modern medicine or even better? The question is disturbing, because it sheds light on the particular status of our knowledge in natural science and medicine: between observation and explanation, there is space for a lot of imagination, which in turn may produce completely different theories. Applying divergent theories to a given problem sometime lead to equivalent results. Examples in science are: geometric and algebric demonstrations for the same theorem; the particle and wave aspects of quantum theory, both of which can predict some phenomena correctly. In medicine, the history of the promising ziconotide, a synthetic peptide coming from snail venom, can be traced back into the antiquity: under very different conceptions of natural sciences, the properties of snails and snail products, were already observed in the time of hippocrates (8), as were those of natural sponges (9).

\section{Conclusion}

Why have some medical theories and systems survived for millennia? In our Mauritanian experience, both medical theories (modern biomedical and classical Greco-Arab) appear similar in their ability to correctly predict an individual's clinical outcome. This may give us a clue of how far we must be, in both medicines, from a unified underlying theory - if such a thing exists within the complexities of human disease and suffering.

One factor in the survival of a given medical system may be its ability to maintain patient confidence, and one important element of that confidence could be the prognostic ability of the practitioner, and in particular the ability to avoid raising false hopes. This study shows that practitioners of different medical systems can correctly predict patient progress. The prognosis/followup method provides a common language for describing 'patient progress', which can be understood by any practitioner and any patient. The practitioner's prognosis ability appears as a key competence, because it allows choosing the best between treatment alternatives, advising referral when necessary and avoiding false expectations. Prognosis and outcome studies such as the one presented here may help to develop the knowledge and the institutions we need: institutions where patients find support in making their choices, not only among several treatment options, but also among several medical systems.

\section{Acknowledgements}

This survey was supported by Médecins du MondeSwitzerland and the Swiss Cooperation Agency, in partnership with the Mauritanian organisation Stand-up Solidarité. We wish to thank the Ould Maqari family and the team and patients of their traditional clinic; Anne Bourgey, Bernard Lambert, Fadil Abdhoum from Médecins du Monde, Switzerland; and we hope that others will forgive us for not naming everyone personally.

\section{References}

1. Bellakhdar J. Médecine Arabe Ancienne et Savoirs Populaires. La Pharmacopée Marocaine Traditionnelle. Paris: Ibis Press, 1997.

2. De Groulard M. Pratiques de médecine traditionnelle et planification sanitaire (exemple de médecine traditionnelle dans l'Adrar Mauritanien). Médecine tropicale 1991;51:37-41.

3. Israël L. La Décision Médicale. Paris: Calamann-Lévi, 1980. 
4. Graz B, Falquet J, Morency P. Assessment of alternative medicine through a comparison of the expected and observed progress of patients: a feasibility study of the prognosis/follow-up method. J Altern Complem Med 2003;9:755-61.

5. Unden AL, Elofsson S. Health from the patient's point of view. How does it relate to the physician's judgement? Family Practice 2001;18:174-80.

6. Lee Y. The predictive value of self assessed general, physical, and mental health on functional decline and mortality in older adults. J Epidemiol Commun Health 2000;54:123-9.

7. Poses RM, Bekes C, Copare FJ, Scott WE. The answer to "What are my chances, Doctor ?" depends on whom is asked: prognostic disagreement and inaccuracy for critically ill patients. Crit Care Med 1989;17:827-33

8. Bruno B. Helix and drugs: snails for Western health care from antiquity to the present. Evid Based Complement Alternat Med 2005;2:25-28. (http://ecam.oxfordjournals.org/cgi/reprint/2/1/25).

9. Müller WE, Batel R, Schröder HC, Müller IM. Traditional and modern biomedical prospecting: part i-the history: sustainable exploitation of biodiversity (sponges and invertebrates) in the Adriatic Sea in Rovinj (Croatia). EvidBased Complement Alternat Med 2004;1:71-82.

Received June 28, 2007; accepted February 21, 2008 


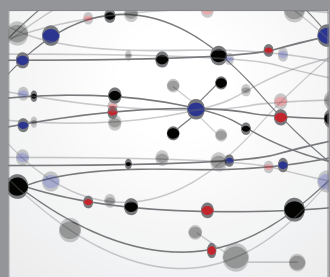

The Scientific World Journal
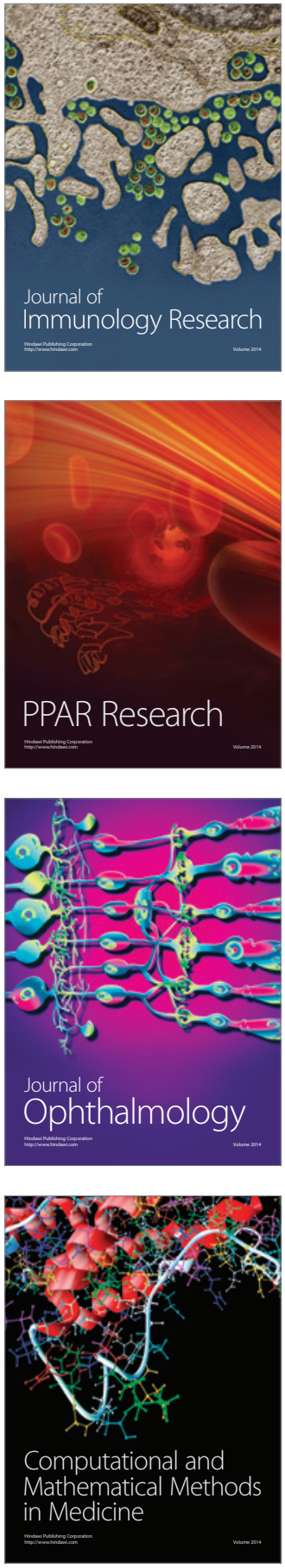

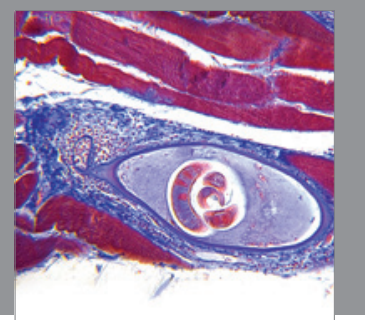

Gastroenterology

Research and Practice
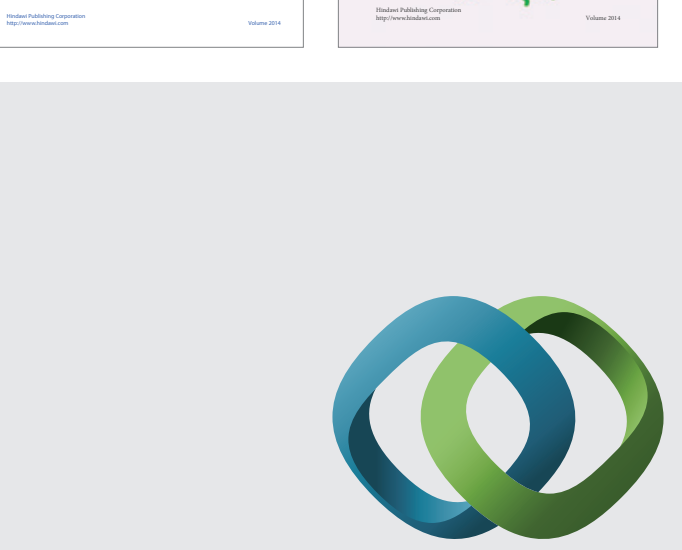

\section{Hindawi}

Submit your manuscripts at

http://www.hindawi.com
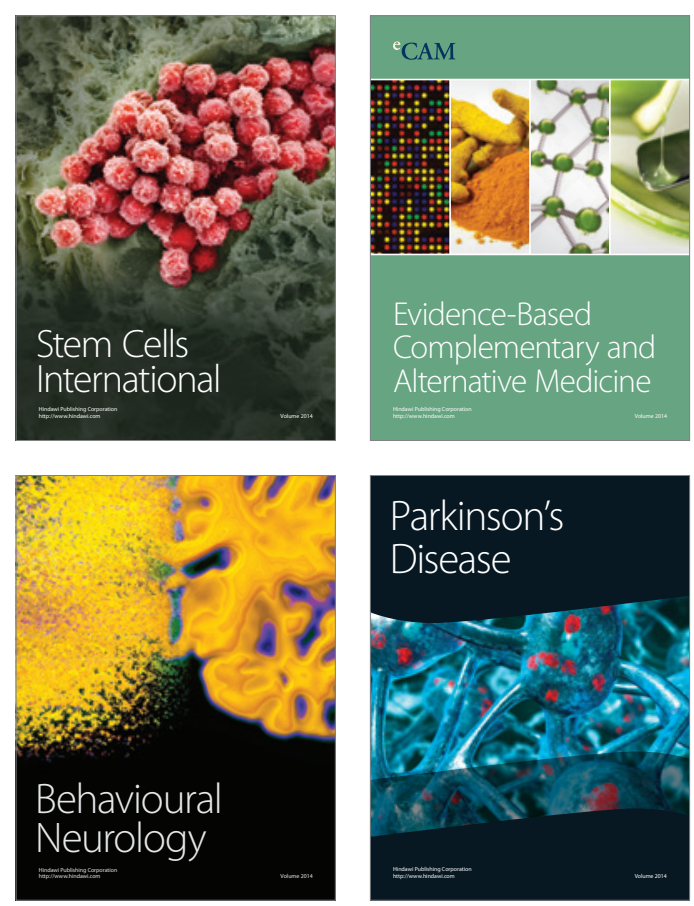

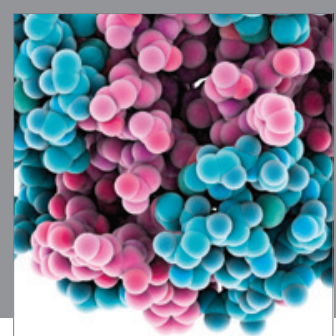

Journal of
Diabetes Research

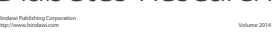

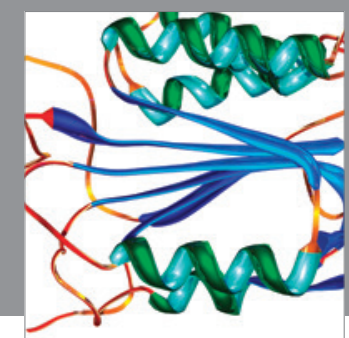

Disease Markers
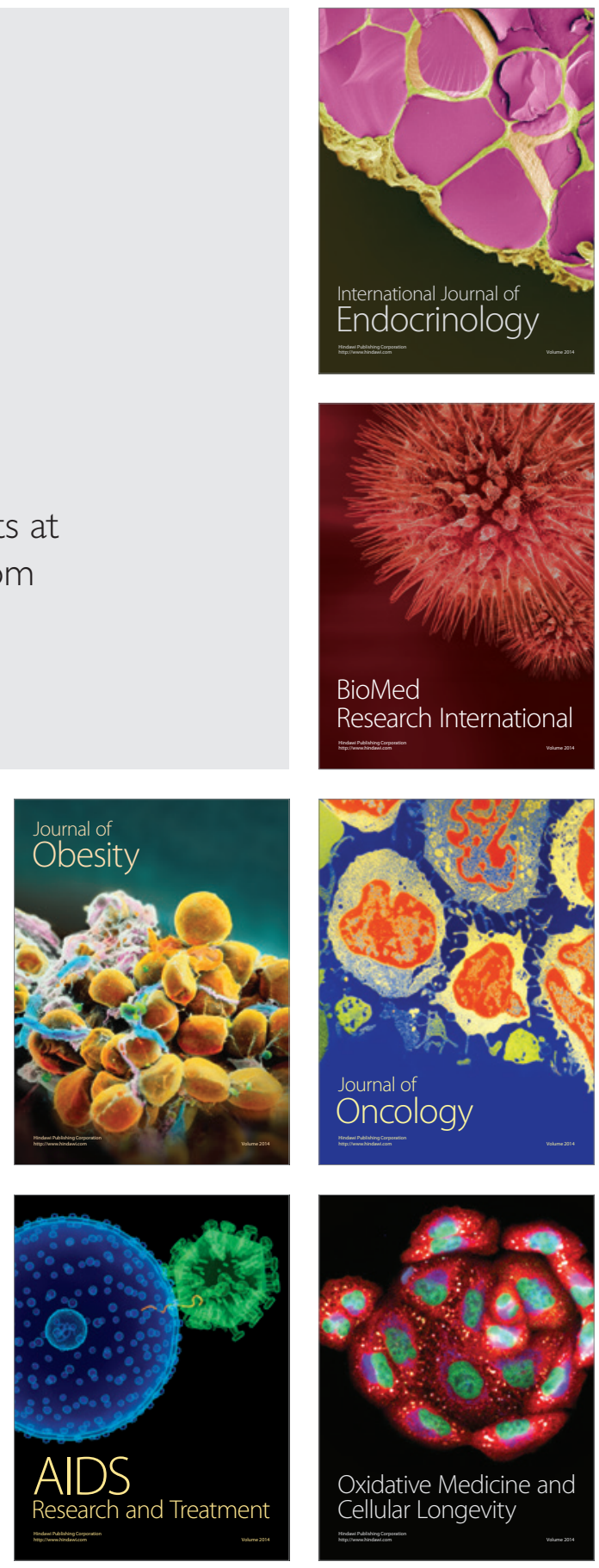